\title{
APPROXIMATE STOLZ ANGLE LIMITS
}

\section{E. SNYDER}

1. Introduction. In a recent paper [4] the author discussed functions of a real variable that are obtained as Soltz angle limits of functions of two real variables. The central idea of this paper is the weakening of the hypothesis of existence of limits with respect to Stolz angles to existence of approximate limits with respect to Stolz angles. A consequence of these investigations is another proof of the fact that an approximate derivative is in the first Baire class. This was proved by Tolstoff [5] in 1938 and more recently Goffman and Neugebauer [2] gave a less involved proof than that of Tolstoff.

2. Definitions and notation. By a Stolz angle we mean an angular sector in the upper half-plane with its vertex on the $x$-axis. If $S$ is such a Stolz angle and $r$ a positive number, then $S^{r}$ denotes the set of points in $S$ which lie on or below the line $y=1 / r .|E|$ denotes either the 1-dimensional or 2-dimensional Lebesgue measure of the set $E$. It will be clear from the context which is intended.

Let $S_{x}$ be a Soltz angle with vertex $(x, 0)$ and let $E$ be a measurable set in the upper half-plane. The lower metric density of $E$ at $(x, 0)$ relative to $S_{x}$ is

$$
\liminf _{r \rightarrow \infty} \frac{\left|S_{x}^{r} \cap E\right|}{\left|S_{x}^{r}\right|} .
$$

If this number is 1 , the point $(x, 0)$ is said to be a point of density of $E$ relative to $S_{x}$.

A function $f$ of one real variable is called a boundary function of a function $\phi$ defined on the upper half-plane if for each $x, f(x)$ is the limit of $\phi(u, v)$ as $(u, v)$ approaches $(x, 0)$ relative to some set in the upper half-plane with $(x, 0)$ as a limit point.

The symbol $\omega(x, f)$ denotes the oscillation of the function $f$ at the point $x$.

The symbol $l(x, \theta)$ denotes the half-line from the point $(x, 0)$ whose angle of inclination is $\theta . R$ denotes the reals and $W$ denotes the open upper half-plane.

3. Main theorem. In this section $S_{x}$ is used to denote the Stolz angle with vertex $(x, 0)$ which consists of the angular sector between $l(x, \pi / 4)$ and $l(x, 3 \pi / 4)$.

Received by the Editors July 6, 1965 . 
THEOREM 1. Suppose $\phi: W \rightarrow R$ and that for each $x \in R$ there is a set $E_{x} \subset W$ such that

(i) $(x, 0)$ is a point of density of $E_{x}$ relative to $S_{x}$ and

(ii) $\lim \phi(u, v)$ exists as $(u, v) \rightarrow(x, 0)$ relative to the set $E_{x}$, for each $x \in R$. Then the boundary function of $\phi$ determined by the family of sets $\left\{E_{x}\right\}$ is in the first Baire class.

Proof. Let $f$ denote the boundary function. For the proof we show that for any nonempty perfect set $P, f \mid P$ has a point of continuity so that by Baire's theorem [3], $f$ is in the first Baire class.

Suppose $P$ is a nonempty perfect set such that $f \mid P$ has no point of continuity. Let $D_{n}=\{x \in P: \omega(x, f \mid P) \geqq 1 / n\}$. It follows that $P=\cup_{n=1}^{\infty} D_{n}$. Since each $D_{n}$ is closed and $P$ is of the second category in itself, there is an $n_{0}$ and an open interval $I$ such that $I \cap P \neq \varnothing$ and $D_{n_{0}}$ contains $I \cap P$. Let $Q=\operatorname{cl}(I \cap P)$. The set $Q$ is perfect and $\omega(x, f \mid Q) \geqq 1 / n_{0}$ on $I \cap P$.

Let $E_{x}^{n}=S_{x}^{n} \cap E_{x}$, where $S_{x}^{n}$ is the set $\left\{(u, v) \in S_{x}: v \leqq 1 / n\right\}$. For each positive integer $k$, let $A_{k}$ be the set defined by

$$
\begin{aligned}
& A_{k}=\left\{x \in Q:\left|E_{x}^{n}\right|>7 / 8 \cdot\left|S_{x}^{n}\right| \text { for } n \geqq k\right. \\
& \left.\qquad \text { and } p \in E_{x}^{k} \Rightarrow|\phi(p)-f(x)|<1 / M\right\},
\end{aligned}
$$

where $M=16 n_{0}$. Note that $A_{k} \subset A_{k+1}$. It also follows that $Q=\cup_{k=1}^{\infty} A_{k}$ since each point $(x, 0)$ is a point of density of $E_{x}$ relative to $S_{x}$ and $f(x)$ is the boundary limit of $\phi$ relative to $E_{x}$. Since $Q$ is of the second category in itself, there is an integer $q$ and an open interval $J$ such that $J \cap Q \neq \varnothing$ and $A_{q}$ is dense in $J \cap Q$.

Let $x_{0}$ be a fixed point in $A_{q} \cap J$. Note that if $\left|x_{0}-x\right|<1 / q$, then $\left|S_{x_{0}}^{q} \cap S_{x}^{q}\right|>1 / 4 q^{2}=\left|S_{x_{0}}^{q}\right| / 4$. Thus if $x \in A_{q} \cap\left(x_{0}-1 / q, x_{0}+1 / q\right)$, then $E_{x_{0}}^{q} \cap E_{x}^{a} \neq \varnothing$.

Let $\left\{x_{j}\right\}$ be any sequence of points in $Q$ with $x_{0}$ as limit. There is an integer $K$ such that $j>K$ implies that $\left|x_{0}-x_{j}\right|<1 / q$ and $x_{j} \in J$. Fix such a $j$ and let $r=\max \left\{q, k\left(x_{j}\right)\right\}$, where $k\left(x_{j}\right)$ is the smallest positive integer $k$ such that $x_{j} \in A_{k}$. Since $A_{q}$ is dense in $J \cap Q$, there is a point $y$ in $A_{q} \cap J$ such that $\left|y-x_{j}\right|<1 / r$. It follows that $E_{y}^{r} \cap E_{x_{j}}^{r}$ $\neq \varnothing$ and $E_{v}^{q} \cap E_{x_{0}}^{q} \neq \varnothing$. Let $\xi \in E_{\nu}^{r} \cap E_{x_{j}}^{r}$ and $\eta \in E_{v}^{q} \cap E_{x_{0}}^{q}$. Then

$$
\begin{aligned}
\left|f\left(x_{j}\right)-f\left(x_{0}\right)\right| \leqq & \left|f\left(x_{j}\right)-\phi(\xi)\right|+|\phi(\xi)-f(y)| \\
& +|f(y)-\phi(\eta)|+\left|\phi(\eta)-f\left(x_{0}\right)\right| \\
< & 4 / M \\
= & 1 / 4 n_{0} .
\end{aligned}
$$

But this implies that $\omega\left(x_{0}, f \mid Q\right) \leqq 1 / 2 n_{0}$ which is a contradiction. 
Therefore $f \mid P$ has a point of continuity for any nonempty perfect set $P$.

4. Noncongruent Stolz angles. A function $f: R \rightarrow R$ is an honorary function of the second class if there is a function $g$ in the first Baire class such that $f(x)=g(x)$ except on a countable set. (This was introduced by Bagemihl and Piranian [1].)

In Theorem 1 all of the Stolz angles were congruent and each was symmetric about the vertical line through its vertex. This seems to be a severe restriction and, as we shall see later, is more than is necessary. However, if we drop the requirement that the Stolz angles be congruent and require only that each Stolz angle $S_{x}$ be symmetric about the half-line $l(x, \pi / 2)$, then the boundary function may be in the second Baire class even though the function $\phi$ be continuous. This is illustrated by the following theorem.

THEOREM 2. If $f: R \rightarrow R$ is any honorary function of the second Baire class, then there is a continuous function $\phi: W \rightarrow R$ such that $f$ is a boundary function of $\phi$ obtained by approximate limits relative to Stolz angles $S_{x}$ symmetric about the half-lines $l(x, \pi / 2)$.

Proof. Let $g: R \rightarrow R$ be a function in the first Baire class which is equal to $f$ except for a countable set. By a theorem in [4], there is a continuous function $\varphi: W \rightarrow R$ such that $\lim \varphi(u, v)=g(x)$ as $(u, v)$ $\rightarrow(x, 0)$ relative to any Stolz angle with vertex $(x, 0)$, i.e., for any nontangential approach to $(x, 0)$, the limit of $\varphi$ exists and is equal to $g(x)$. Let $\left\{r_{n}\right\}$ be a sequential ordering of the exceptional points. For each $x$, let $S_{x}$ denote the Stolz angle which has as its sides the halflines $l(x, \pi / 4)$ and $l(x, 3 \pi / 4)$. For $x=r_{n}$, let $T_{x}$ be a Stolz angle which is symmetric about $l(x, \pi / 2)$ and satisfies $\left|T_{x}^{a}\right|=\left|S_{x}^{a}\right| \cdot\left(1 / 2^{n}\right)$ for every positive real number $a$. For each $n=1,2, \cdots$, it is possible to truncate $T_{r_{n}}$ at some height, say $1 / k$, so that $T_{r_{n}}^{k} \cap \bigcup_{i=1}^{n-1} T_{r_{i}}$ is empty. Let us suppose that this has been done for each $x=r_{n}$ and let us denote the truncated Stolz angles by $T_{x}$ likewise. Next let $\widetilde{T}_{x}$ be a truncated Stolz angle contained in the interior of $T_{x}$ except that $T_{x}$ and $\widetilde{T}_{x}$ have their vertex in common.

We define $\phi: W \rightarrow R$ as follows: let $\phi(u, v)=\varphi(u, v)$ for any point $(u, v)$ not in $\bigcup_{n=1}^{\infty}$ Int $\left(T_{r_{n}}\right)$, let $\phi(u, v)=f(x)$ for every point in $\tilde{T}_{x}$ $\left(x=r_{n}\right.$ for some $\left.n\right)$, and extend continuously over the remainder of $T_{r_{n}}$ for each $n$.

For $x=r_{n}$, the set $E_{x}$ on which $\phi$ has the proper boundary limit is the set $\widetilde{T}_{x}$. Then clearly $(x, 0)$ is a point of density of $E_{x}$ relative to the Stolz angle $\tilde{T}_{x}$. For a point $x$ which is not one of the $r_{n}$ 's, the set 
$E_{x}$ is $S_{x}$ minus the union of the $T_{r_{n}}$ 's. It is clear from the definition of $\phi$ that $\phi$ has the proper boundary limit relative to the set $E_{x}$. It only remains to verify that $(x, 0)$ is a point of density of $E_{x}$ relative to $S_{x}$. Let $\epsilon>0$ be given. There is an integer $N$ such that $n>N$ implies $\sum_{k=1}^{\infty} 1 / 2^{k}<\epsilon$. Let $M$ be chosen so large that $m \geqq M$ implies that $S_{x}^{m} \cap \bigcup_{n=1}^{N} T_{r_{n}}=\varnothing$. For $m \geqq M$,

$$
\begin{aligned}
\frac{\left|E_{x}^{m}\right|}{\left|S_{x}^{m}\right|} & =\frac{\left|S_{x}^{m}-\bigcup_{n=N+1}^{U} T_{r_{n}}^{m}\right|}{\left|S_{x}^{m}\right|} \geqq 1-\sum_{n=N+1}^{\infty} \frac{\left|T_{r_{n}}^{m}\right|}{\left|S_{x}^{m}\right|} \\
& =1-\sum_{n=N+1}^{\infty} \frac{1}{2^{n}}>1-\epsilon .
\end{aligned}
$$

This verifies the density of $E_{x}$ at $(x, 0)$ relative to $S_{x}$ and completes the proof.

In [4] it was shown that the class of functions which are Stolz angle boundary functions is a proper subclass of the class of honorary functions of the second Baire class. Here we note that if a function is an approximate Stolz angle boundary function, with the Stolz angles being suitably restricted, then the function is in the first Baire class, whereas if the restrictions on the Stolz angles are slightly weakened, then any honorary function can be realized as the approximate Stolz angle boundary function of a continuous function.

5. Corollaries of Theorem 1. For each $x \in R$, let $S_{x}$ again denote the Stolz angle whose sides are $l(x, \pi / 4)$ and $l(x, 3 \pi / 4)$. In the following corollary it is shown that the hypothesis on the $E_{x}$ 's can be weakened without changing the conclusion of Theorem 1.

CoRollary 1. Theorem 1 remains valid if in place of (i) it is only required that there is a constant $K>1 / 2$ such that the lower metric density of $E_{x}$ at $(x, 0)$ relative to $S_{x}$ is greater than or equal to $K$.

In order to prove Corollary 1 it is only necessary to make a few modifications in the proof of Theorem 1. For example, define $A_{k}$ by

$$
\begin{aligned}
& A_{k}=\left\{x \in Q:\left|E_{x}^{n}\right|>\zeta \cdot\left|S_{x}^{n}\right| \text { for } n \geqq k \text { and } p \in E_{x}^{k} \Rightarrow\right. \\
& \qquad|\phi(p)-f(x)|<1 / M\},
\end{aligned}
$$

where $1 / 2<\zeta<K$ and $M=16 n_{0}$. At a later step, assuming $q$ has been selected as before, it is necessary to find a positive number $\delta$ so that $\left|x_{0}-x\right|<\delta$ implies $\left|S_{x_{0}}^{q} \cap S_{x}^{q}\right|>2 \cdot(1-\eta) \cdot\left|S_{x_{0}}^{q}\right|$. From the previous inequality it follows that $E_{x_{0}}^{q} \cap E_{x}^{q} \neq \varnothing$ for $x \in A_{q} \cap\left(x_{0}-\delta, x_{0}+\delta\right)$. The remainder of the proof is essentially the same. 
Another direction to proceed in considering weakenings of the hypotheses of Theorem 1 is to allow more general Stolz angles $S_{x}$, that is, to allow the "size" of $S_{x}$ to vary with $x$. Of course, as can be seen in $\S 4$, it will be necessary to impose some restrictions on the varying in order to obtain the same conclusion as in Theorem 1.

We will continue to consider only Stolz angles $S_{x}$ that are symmetric about the line $l(x, \pi / 2)$. Let us suppose that a family of Stolz angles $\left\{S_{x}: x \in R\right\}$ is given. Let $\theta(x)$ denote the angle between the sides of $S_{x}$. Then corresponding to the family $\left\{S_{x}: x \in R\right\}$ there is a function $\theta: R \rightarrow(0, \pi)$.

Corollary 2. Suppose $\phi: W \rightarrow R$ and that for each $x \in R$ there is a set $E_{x} \subset W$ such that

(i) $(x, 0)$ is a point of density of $E_{x}$ relative to $S_{x}$ and

(ii) $\lim \phi(u, v)$ exists as $(u, v) \rightarrow(x, 0)$ relative to the set $E_{x}$, for each $x \in R$. If the function $\theta: R \rightarrow(0, \pi)$ associated with the family of Stolz angles is upper semicontinuous, then the boundary function $f$ of $\phi$ determined by the family of sets $E_{x}: X \in R$ is in the first Baire class.

Proof. Suppose that there is a nonempty perfect set $P$ for which $f \mid P$ has no point of continuity. For each $n=1,2, \cdots$, let $B_{n}$ $=\{x \in P: \theta(x) \geqq 1 / n\}$. Since $\theta$ is upper semicontinuous, each $B_{n}$ is closed. Also $P=\cup_{n=1}^{\infty} B_{n}$. Since $P$ is second category in itself, there is an integer $n_{1}$ and an open interval $J$ such that $B_{n_{1}}$ is dense in $J \cap P$ and $J \cap P \neq \varnothing$. From this it follows that $B_{n_{1}} \supset J \cap P$. Then $\theta(x)$ $\geqq 1 / n_{1}$ for $x \in J \cap P$. Then for each $x \in J \cap P$, let $T_{x}$ be a symmetric Stolz angle with vertex at $(x, 0)$ and with the angle between its sides being $1 / n_{1}$. The point $(x, 0)$ is also a point of density of $E_{x}$ relative to $T_{x}$.

The remainder of the proof consists of showing that $f \mid P$ has a point of continuity in $J \cap P$. This is accomplished by the same means used in the proof of Theorem 1, using the $T_{x}$ 's instead of the original $S_{x}$ 's. We omit the details of this.

6. Approximate derivatives. Let $I_{0}$ be an open interval and let $f: I_{0} \rightarrow R$. The number $A$ is called the approximate derivative of the function $f$ at $x_{0}$ if there exists a set $E$, having $x_{0}$ as a point of metric density one, such that for $x \in E$ and $x \rightarrow x_{0}$ we have

$$
\lim \left(f(x)-f\left(x_{0}\right)\right) /\left(x-x_{0}\right)=A .
$$

In this case we use the symbol $f_{a p}^{\prime}\left(x_{0}\right)$ for the number $A$. In this section we are interested in the function $f_{a p}^{\prime}$ whenever it exists everywhere on some interval. 
THEOREM 3. Let $f: I_{0} \rightarrow R$. If $f^{\prime}{ }_{a p}(x)$ exists for each $x \in I_{0}$, then the function $f_{a p}^{\prime}$ is in the first Baire class.

Proof. For the proof we construct a function $\phi: I_{0} \times(0, \infty) \rightarrow R$ which has $f_{a p}^{\prime}$ as its approximate Stolz angle boundary function (in the sense of Theorem 1$)$.

Let $U$ denote $I_{0} \times(0, \infty)$. If $(x, r) \in U$ and if $x-r / 2$ and $x+r / 2$ are both in $I_{0}$, let $\phi(x, r)=(f(x+r / 2)-f(x-r / 2)) / r$. If $(x, r) \in U$ and if either $x-r / 2$ or $x+r / 2$ is not in $I_{0}$, let $\phi(x, r)=0$.

Let $x_{0} \in I_{0}$ be fixed. We will show that there is a set $E_{x_{0}} \subset U$ such that $\left(x_{0}, 0\right)$ is a point of density of $E_{x_{0}}$ relative to $S_{x_{0}}$ and such that $\lim \phi(x, r)=f\left(x_{0}\right)$ for $(x, r) \in E_{x_{0}}$ and $(x, r) \rightarrow\left(x_{0}, 0\right)$. By hypothesis there is a set $B_{x_{0}} \subset I_{0}$ such that the linear density of $B_{x_{0}}$ at $x_{0}$ is 1 and $\lim \left(f(x)-f\left(x_{0}\right)\right) /\left(x-x_{0}\right)=f_{a p}^{\prime}\left(x_{0}\right)$ for $x \in B_{x_{0}}$ and $x \rightarrow x_{0}$. Let $E_{x_{0}}$ be the set defined by $E_{x_{0}}=\left\{(x, r) \in U: x_{0}-r / 2 \leqq x \leqq x_{0}+r / 2, x+r / 2\right.$ $\in B_{x_{0}}$, and $\left.x-r / 2 \in B_{x_{0}}\right\}$, and let $\left\{\left(x_{n}, r_{n}\right)\right\}$ be a sequence of points in $E_{x_{0}}$ with $\left(x_{0}, 0\right)$ as limit. We have

$$
\begin{aligned}
& \left|\phi\left(x_{n}, r_{n}\right)-f_{a p}^{\prime}\left(x_{0}\right)\right|=\left|\frac{f\left(x_{n}+r_{n} / 2\right)-f\left(x_{n}-r_{n} / 2\right)}{r_{n}}-f_{a p}^{\prime}\left(x_{0}\right)\right| \\
& =\mid\left(\frac{f\left(x_{n}+r_{n} / 2\right)-f\left(x_{0}\right)}{x_{n}+r_{n} / 2-x_{0}}-f_{a p}^{\prime}\left(x_{0}\right)\right) \cdot\left(\frac{x_{n}+r_{n} / 2-x_{0}}{r_{n}}\right) \\
& +\left(\frac{f\left(x_{0}\right)-f\left(x_{n}-r_{n} / 2\right)}{x_{0}-x_{n}+r_{n} / 2}-f_{a p}^{\prime}\left(x_{0}\right)\right) \cdot\left(\frac{x_{0}-x_{n}+r_{n} / 2}{r_{n}}\right) \mid \\
& \leqq\left|\frac{f\left(x_{n}+r_{n} / 2\right)-f\left(x_{0}\right)}{x_{n}+r_{n} / 2-x_{0}}-f_{a p}^{\prime}\left(x_{0}\right)\right|+\left|\frac{f\left(x_{0}\right)-f\left(x_{n}-r_{n} / 2\right)}{x_{0}-x_{n}+r_{n} / 2}-f_{a p}^{\prime}\left(x_{0}\right)\right| \text {. }
\end{aligned}
$$

The two expressions on the extreme right of the inequality approach zero as $n \rightarrow \infty$ since $x_{n}-r_{n} / 2$ and $x_{n}+r_{n} / 2$ are in $B_{x_{0}}$. Therefore $\phi\left(x_{n}, r_{n}\right) \rightarrow f_{a p}^{\prime}\left(x_{0}\right)$.

It remains to verify the density property of $E_{x_{0}}$. For this end, let $\epsilon$ be an arbitrary positive number. Since $B_{x_{0}}$ has $x_{0}$ as a point of density, there is a number $R(\epsilon)$ such that $r<R(\epsilon)$ implies that $\left|B_{x_{0}} \cap\left(x_{0}-r, x_{0}+r\right)\right|>(1-\epsilon) \cdot 2 r$. Let $r$ less than $R(\epsilon)$ be fixed, and let $\eta$ denote the linear Lebesgue measure of the intersection of $E_{x_{0}}$ with the line $y=r / 2$. Let $D=\left\{x \in\left(x_{0}-r / 2, x_{0}+r / 2\right): x+r / 2 \in B_{x_{0}}\right.$ and $\left.x-r / 2 \in B_{x_{0}}\right\}$, then $\eta=|D|$. Let $F=\left(x_{0}-r / 2, x_{0}+r / 2\right) \sim D$. If $x \in F$, then $x+r / 2$ or $x-r / 2$ is not in $B_{x_{0}}$. But the points $x+r / 2$ and $x-r / 2$ lie in the interval $\left(x_{0}-r, x_{0}+r\right)$ and the measure of the set of 
points in $\left(x_{0}-r, x_{0}+r\right)$ which are not in $B_{x_{0}}$ is less than $\epsilon \cdot 2 r$. Thus the measure of $F$ is no more than $\epsilon \cdot 4 r$, and so $\eta>(1-2 \epsilon) \cdot 2 r$. It now follows that for $r<R(\epsilon)$, the relative measure of $B_{x_{0}}$ in $S_{x_{0}}$, truncated at $y=r$, is greater than or equal to $(1-2 \epsilon)$ times the measure of the truncated Stolz angle. This completes the proof.

REMARK. Theorem 1 also implies the well-known fact that approximately continuous functions are in the first Baire class. For if $f: R \rightarrow R$ is approximately continuous, let $\phi(x, y)=f(x)$. Clearly $f$ is an approximate Stolz angle boundary function for $\phi$ in the sense of Theorem 1.

In the theorems above, sets with zero density at certain points were the exceptional sets. Analogous theorems are true if one considers first category sets as the exceptional sets.

\section{REFERENCES}

1. F. Bagemihl and G. Piranian, Boundary functions for functions defined in a disk, Michigan Math. J. 8 (1961), 201-207.

2. C. Goffman and C. J. Neugebauer, On approximate derivatives, Proc. Amer. Math. Soc. 11 (1960), 962-966.

3. F. Hausdorff, Set theory (Transl. 3rd ed. of Mengenlehre, 1937), Chelsea, New York, 1957, 288-289.

4. L. Snyder, Continuous Stolz extensions and boundary functions, Trans. Amer. Math. Soc. 119 (1965), 417-427.

5. G. Tolstov, Sur la dérivée approximative exact, Rec. Math. (Mat. Sb. (N.S.)) 4 (1938), 499-504.

Purdue University 